\title{
Clinical impacts of inflammatory markers and clinical factors in patients with relapsed or refractory diffuse large B-cell lymphoma
}

\author{
Do-Young Kim ${ }^{1 \#}$, Moo-Kon Song ${ }^{2 *}$, Joo-Seop Chung ${ }^{1}$, Ho-Jin Shin ${ }^{1}$, Deok Hwan Yang ${ }^{3}$, \\ Sung-Nam Lim ${ }^{4}$, Sung-Yong $\mathrm{Oh}^{5}$ \\ Department of Hematology-Oncology, ${ }^{1}$ Pusan National University Hospital Medical Research Institute, Busan, ${ }^{2}$ Hanyang University \\ Hanmaeum Changwon Hospital, Changwon, Department of Hematology, ${ }^{3}$ Chonnam National University Hwasun Hospital, Hwasun, \\ ${ }^{4}$ Busan Haeundae Paik Hospital, ${ }^{5}$ Dong-A University Hospital, Busan, Korea
}

\begin{abstract}
p-ISSN 2287-979X / e-ISSN 2288-0011 https://doi.org/10.5045/br.2019.54.4.244 Blood Res 2019;54:244-252.
\end{abstract}

Received on May 29, 2019

Revised on August 9, 2019

Accepted on August 29, 2019 \#These authors contributed equally to this
work.

\section{*This study was supported by a 2-year research grant from the Pusan National University. \\ Correspondence to \\ Joo-Seop Chung, M.D., Ph.D. \\ Department of Hematology-Oncology, \\ Pusan National University Hospital \\ Medical Research Institute, 179 \\ Gudeok-ro, Seo-gu, Busan 49241, Korea \\ E-mail: hemon@pusan.ac.kr \\ (C) 2019 Korean Society of Hematology}

\section{Background}

Systemic inflammatory response can be associated with the prognosis of diffuse large B cell lymphoma (DLBCL). We investigated the systemic factors significantly related to clinical outcome in relapsed/refractory DLBCL.

\section{Methods}

In 242 patients with DLBCL, several factors, including inflammatory markers were analyzed. We assessed for the correlation between the survivals [progression-free survival (PFS) and overall survival (OS)] and prognostic factors.

\section{Results}

In these patients, a high derived neutrophil/lymphocyte ratio ( $d N L R$ ) (PFS, HR=2.452, $P=0.002$; OS, $\mathrm{HR}=2.542, P=0.005)$, high Glasgow Prognostic Score (GPS) (PFS, $\mathrm{HR}=2.435, P=0.002 ; \mathrm{OS}, \mathrm{HR}=2.621, P=0.002)$, and high NCCN-IPI (PFS, HR=2.836, $P=0.003$; OS, $\mathrm{HR}=2.928, P=0.003$ ) were significantly associated with survival in multivariate analysis. Moreover, we proposed a risk stratification model based on dNLR, GPS, and NCCN-IPI, thereby distributing patients into 4 risk groups. There were significant differences in survival among the 4 risk groups (PFS, $P<0.001$; OS, $P<0.001$ ).

\section{Conclusion}

In conclusion, dNLR, GPS, and NCCN-IPI appear to be excellent prognostic parameters for survival in relapsed/refractory DLBCL.

Key Words Derived neutrophil/lymphocyte ratio, Glasgow prognostic score, DLBCL

\section{INTRODUCTION}

Diffuse large B cell lymphoma (DLBCL) is the most common lymphoid malignancy, accounting for $25-30 \%$ of all the newly diagnosed cases of adult non-Hodgkin's lymphoma (NHL) [1]. Despite an improvement in the overall survival of patients with DLBCL after the introduction of rituximab, cyclophosphamide, doxorubicin, vincristine, and prednisone (R-CHOP) therapy into clinics, one-third of the patients remain refractory to the initial therapy or relapse afterward [2]. Therefore, it would be beneficial to identify prognostic markers for the prediction of any subgroups with a poor prognosis within the patients with relapsed or refractory DLBCL. In the Collaborative Trial in Relapsed Aggressive
Lymphoma (CORAL) study, an early relapse $(<1 \mathrm{yr})$ after diagnosis, previous exposure to rituximab, and the age-adjusted International Prognostic Index (IPI) were demonstrated to be significant prognostic parameters associated with the survival rate of patients with relapsed or refractory DLBCL [3]. Recently, the National Comprehensive Cancer Network (NCCN)-IPI has been introduced as a more meaningful prognostic parameter than the traditional IPI for newly diagnosed DLBCL cases [4]. A recent study has shown that a higher NCCN-IPI is significantly associated with a low overall response rate and poor survival in relapsed or refractory DLBCL [5]. However, further research is needed to confirm whether NCCN-IPI specifies clinical outcomes in the relapsed or refractory setting.

Furthermore, cell of origin (COO) subtype in relapsed 
or refractory DLBCL has also been noticed as a prominent prognostic biomarker for survival prediction since DLBCL cases differ in their cellular compositions [5]. However, gene expression profiling or immunohistochemical analysis to determine $\mathrm{COO}$ has not been widely implemented in clinical practice.

There seems to be more solid evidence from several studies that inflammation is closely associated with the pathogeneses of NHLs [6-8]. Pro-inflammatory cytokines in tumor microenvironment have been shown to promote tumor growth, DNA damage, angiogenesis, and immune suppression [9-11]. Therefore, inflammation could negatively affect clinical outcome in NHLs. Indeed, among the various inflammatory markers associated with cancer, cell-based inflammatory markers including neutrophil/lymphocyte ratio (NLR), derived NLR (dNLR), lymphocyte/monocyte ratio (LMR), and platelet/lymphocyte ratio (PLR) have been associated with survival in newly diagnosed or relapsed/refractory DLBCL [12-17]. The nutrition-related inflammatory markers Glasgow Prognostic Score (GPS) and Prognostic Nutritional Index (PNI) have also been associated with a poor prognosis in the newly diagnosed DLBCL patients $[18,19]$. However, to our best knowledge, it is still unclear what could be the most useful parameter among the above-mentioned inflammatory markers and clinical factors, such as IPI and NCCN-IPI, to predict survival in relapsed or refractory DLBCL. Moreover, there is still no clearly established risk stratification model to predict survival in relapsed or refractory DLBCL, although various prognostic factors have been extensively described in the relapsed or refractory setting.

Therefore, this study aimed to assess what inflammatory marker could be the most meaningful prognostic factor for predicting disease progression and survival in patients with relapsed or refractory DLBCL. Furthermore, we attempted to define a prognostic model that incorporates the significant factors associated with patients with relapsed or refractory DLBCL.

\section{MATERIALS AND METHODS}

\section{Patient eligibility}

The information about the patients who had had relapsed after R-CHOP therapy as the first-line therapy and who progressed during the initial therapy from January 2007 to September 2016 was collected. In this study, a relapsed or refractory disease was defined according to the criteria outlined by Cheson et al. [20]. Patients were excluded if they had chronic diseases, such as chronic renal disease, chronic hepatitis $\mathrm{B}$ and $\mathrm{C}$, and pulmonary tuberculosis because they could influence the initial levels of C-reactive protein (CRP) and albumin in the serum and negatively affect the disease management or survival of the patients. Patients were also excluded if they presented with DLBCL secondary to low-grade NHL or had received other follow-up treatments, including maintenance therapy and radiotherapy after
R-CHOP therapy. Eventually, 242 patients diagnosed with relapsed or refractory DLBCL after or during first-line $\mathrm{R}$-CHOP therapy were enrolled. Relapsed cases in central nervous system were excluded. Patients who achieved complete or partial response after salvage chemotherapy entered either follow-up or autologous stem cell transplantation (ASCT). Patients who did not achieve any response were treated with supportive care.

\section{Ethics statement}

The retrospective review of the records for this study was approved by the Institutional Review Board of five medical centers, including Pusan National University Hospital, Hanyang University Hanmaeum Changwon Hospital, Chonnam National University Hwasun Hospital, Dong-A University Hospital, and Haeundae Paik Hospital.

\section{Salvage treatment and response assessment}

Three salvage chemotherapy schedules were adopted for patients with relapsed/refractory DLBCL. The regimens were as follows: ESHAP/R-ESHAP (etoposide, methylprednisolone, cytarabine, and cisplatin with/without rituximab), DHAP/ R-DHAP (dexamethasone, cisplatin, and cytarabine with/ without rituximab), or ICE/R-ICE (ifosfamide, carboplatin, and etoposide with/without rituximab). Treatment response was assessed using the National Cancer Institute-sponsored Working Group guidelines [20]. After the salvage chemotherapy was completed, the patients were followed-up with physical examinations and laboratory tests for every 3 months over 5 years, and imaging tests were also performed twice/year during the follow-up period.

\section{Prognostic factors}

The serum beta-2 microglobulin (B2MG) level of each patient at the relapsed or refractory status was measured to evaluate whether the level of this protein was a meaningful prognostic marker. In addition, GPS was determined by the serum CRP and albumin levels measured at the time of a patient's presentation with a relapsed or refractory status. Patients with both elevated CRP ( $\geq 10 \mathrm{mg} / \mathrm{L})$ and decreased albumin levels $(<35 \mathrm{~g} / \mathrm{L})$ were classified into GPS 2 group. Patients with only one of these two laboratory abnormalities were classified into GPS 1 group, and patients without these abnormalities were classified into GPS 0 group.

To test the clinical value of GPS, several comparative prognostic factors were included as follows: IPI or NCCN-IPI score of the IPI or NCCN-IPI factor, respectively, at the relapsed/refractory status was included (high IPI and NCCN-IPI were defined as $\geq 3$ and $\geq 5$ scores, respectively). Additional comparative variables, such as the primary refractory type, which further progresses during R-CHOP therapy, and the maximum 18F-fludeoxyglucose uptake value (SUVmax) in positron emission tomography (PET)/computed tomography (CT) measured at the relapsed or refractory status were also included.

As systemic inflammatory factors, NLR, dNLR, LMR, PLR, $\mathrm{PNI}$, systemic inflammation response index (SIRI), and sys- 
temic inflammation index (SII) at the relapsed or refractory status were included. dNLR was defined as neutrophil/(white blood cell count-neutrophil count) at the relapsed or refractory status. PNI was estimated by the following equation:

10×serum albumin (g/dL)+(0.005×total lymphocyte count)

SIRI was defined as follows: peripheral neutrophil countx monocyte count/lymphocyte counts. SII was defined as follows: platelet count $\times$ neutrophil count/lymphocyte count. SIRI score was considered as follows: patients with both elevated hemoglobin $(\mathrm{Hb})$ level and elevated LMR at the relapsed or refractory status $(\geq 137 / 116 \mathrm{~g} / \mathrm{L}$ and $\geq 3.23$, respectively) were considered to have a score of 2 (group 2); patients with either elevated $\mathrm{Hb}$ level or elevated LMR were considered to have a score of 1 (group1); and patients with both decreased $\mathrm{Hb}$ level and decreased LMR were considered to have a score of 0 (group 0).

Additional factors such as male sex, R-containing salvage therapy, B symptom at the relapsed or refractory state, and ASCT after salvage therapy were included to compare with the inflammatory markers. However, the cell of origin determined by immunohistochemistry was excluded in our analysis since such histological assessments were performed in only 81 patients (33.5\%).

\section{Statistical analysis}

Chi-square test or Fisher's exact test were used as appropriate to analyze categorical variables. Progression-free survival (PFS) and overall survival (OS) were estimated using the Kaplan-Meier method and the 2-tailed log-rank test. PFS was defined as the time from the initiation of the salvage therapy until disease progression or death, whereas OS was defined as the time from the initiation of the salvage therapy until death.

The Cox proportional-hazards model was used to evaluate the prognostic impacts of several prognostic factors. The hazard ratios (HRs) of the prognostic factors were used to measure the differential risks of disease progression and death. Receiver operating characteristic (ROC) curves were prepared to estimate the accuracy in predicting the ideal cut-off values of the continuous variables. The statistical analysis was carried out with the SPSS software version 18.0 (SPSS Inc., Chicago, IL, USA). A $P$-value $<0.05$ was considered significant.

\section{RESULTS}

\section{Patient characteristics}

A total of 242 patients with relapsed or refractory DLBCL from five medical centers were evaluated, and their clinical characteristics are summarized in Table 1. Their median age was 65 years (range, $40-76 \mathrm{yr}$ ), and 160 patients $(66.1 \%)$ were $>60$ years old (of these, 154 patients were $\leq 75$ years old and 6 patients were $>75$ years old). The patients included 143 males $(59.1 \%)$ with 45 patients of the primary refractory
Table 1. The baseline characteristics of the patients with relapsed/ refractory diffuse large B cell lymphoma.

\begin{tabular}{|c|c|}
\hline Baseline characteristics & Total $(\mathrm{N}=242)$ \\
\hline \multicolumn{2}{|l|}{ Age } \\
\hline Median (range) & $65(40-79)$ \\
\hline$\leq 60 \mathrm{yr}(\%)$ & $82(33.9)$ \\
\hline$>60$ to $\leq 75 \mathrm{yr}(\%)$ & $154(63.6)$ \\
\hline$>75$ yr $(\%)$ & $6(2.5)$ \\
\hline \multicolumn{2}{|l|}{ Sex } \\
\hline Male (\%) & $143(59.1)$ \\
\hline Female (\%) & 99 (40.9) \\
\hline \multicolumn{2}{|l|}{ Disease progression pattern } \\
\hline Primary refractory type (\%) & $45(18.6)$ \\
\hline \multicolumn{2}{|l|}{ Ann-Arbor stage } \\
\hline Stage I/II (\%) & $119(49.2)$ \\
\hline Stage III/IV (\%) & $123(50.8)$ \\
\hline \multicolumn{2}{|l|}{ Serum LDH level } \\
\hline$>1$ to $\leq 3(\%)$ & $78(32.2)$ \\
\hline$>3(\%)$ & $65(26.9)$ \\
\hline \multicolumn{2}{|l|}{ EN site involvement } \\
\hline$\geq 2$ EN sites (\%) & $49(20.2)$ \\
\hline EN disease $(\%)$ & $63(26.0)$ \\
\hline \multicolumn{2}{|l|}{ ECOG performance status } \\
\hline$\geq$ Grade $2(\%)$ & $41(16.9)$ \\
\hline \multicolumn{2}{|l|}{ B symptoms } \\
\hline Presence of B symptoms (\%) & $98(40.5)$ \\
\hline \multicolumn{2}{|l|}{ B2MG level } \\
\hline Median (range) & $2.65(1.10-9.80)$ \\
\hline \multicolumn{2}{|l|}{ IPI score } \\
\hline Low IPI (0-2) (\%) & $137(56.7)$ \\
\hline High IPI (3-5) (\%) & 85 (43.3) \\
\hline \multicolumn{2}{|l|}{ NCCN-IPI score } \\
\hline Low NCCN-IPI (0-4) (\%) & $159(65.7)$ \\
\hline High NCCN-IPI (5-8) (\%) & $63(34.3)$ \\
\hline \multicolumn{2}{|l|}{ GPS group } \\
\hline GPS 0 group (\%) & $83(34.3)$ \\
\hline GPS 1 group (\%) & $100(41.3)$ \\
\hline GPS 2 group (\%) & $59(24.4)$ \\
\hline \multicolumn{2}{|l|}{ Laboratory finding } \\
\hline ANC $\left(\times 10^{9} / \mathrm{L}\right)$, median (range) & $3.6(0.1-14.4)$ \\
\hline ALC $\left(\times 10^{9} / \mathrm{L}\right)$, median (range) & $1.83(0.33-7.78)$ \\
\hline AMC $\left(\times 10^{9} / \mathrm{L}\right)$, median (range) & $0.44(0.12-1.95)$ \\
\hline $\mathrm{Hb}(\mathrm{g} / \mathrm{L})$, median (range) & $102(80-159)$ \\
\hline PLT $\left(\times 10^{9} / \mathrm{L}\right)$, median (range) & $169(25-528)$ \\
\hline CRP (mg/L), median (range) & $13.0(0.1-153.6)$ \\
\hline Albumin (g/L), median (range) & $39(22-49)$ \\
\hline \multicolumn{2}{|l|}{ PET/CT finding } \\
\hline SUVmax, median (range) & $12.5(3.2-39.5)$ \\
\hline \multicolumn{2}{|l|}{ Salvage chemotherapy } \\
\hline R-containing regimen (\%) & $126(52.1)$ \\
\hline R-ESHAP/ESHAP (\%) & $63(26.0) / 93(38.4)$ \\
\hline R-DHAP/DHAP (\%) & $20(8.3) / 9(3.7)$ \\
\hline R-ICE/ICE (\%) & $43(17.8) / 14(5.8)$ \\
\hline \multicolumn{2}{|l|}{ ASCT } \\
\hline Received numbers (\%) & $102(42.1)$ \\
\hline
\end{tabular}

Abbreviations: ALC, absolute lymphocyte count; AMC, absolute monocyte count; ANC, absolute neutrophil count; ASCT, autologous stem cell transplantation; B2MG, beta-2-microglobulin; CRP, C-reactive protein; CT, computed tomography; ECOG, Eastern Cooperative Oncology Group; EN, extranodal; GPS, glasgow prognostic score; $\mathrm{Hb}$, hemoglobin; IPI, International Prognostic Index; LDH, lactate dehydrogenase; NCCN, National Comprehensive Cancer Network; PET, positron emission tomography; PLT, platelet; SUVmax, maximum standard uptake value. 
type (18.6\%). Additionally, 123 patients (50.8\%) were at the advanced stage (stages III and IV), and 143 patients (59.1\%) had elevated LDH levels [ > one-fold upper normal limit (UNL) but $\leq$ three-fold UNL, N=78 (32.2\%); > three-fold UNL, N=65 (26.9\%)]. Among the patients, 49 (20.2\%) had $\geq 2$ extranodal (EN) site involvement and 63 (26.0\%) had EN disease as an NCCN-IPI factor. Eastern Cooperative Oncology Group (ECOG) performance status (PS) above grade 2 was present in 41 patients $(16.9 \%)$, and 98 patients (40.5\%) had B symptoms.

Based on the above clinical data at the relapsed or refractory status, 85 patients $(43.3 \%)$ had high IPI scores $(\geq 3)$, while 85 patients $(43.3 \%)$ had high NCCN-IPI scores $(\geq 5)$. According to GPS, 59 patients were included in GPS 2 group (24.4\%), while 183 patients were included in other GPS groups (GPS 0, N=83, 34.3\%; GPS 1, N=100, 41.3\%). In PET/CT scans performed at the relapsed or refractory status, the median value of the SUVmax was 12.5 (range, 3.2-39.5). For disease management, 126 patients $(52.1 \%)$ were treated with R-containing regimens and ASCT after salvage therapy was performed in 102 patients (42.1\%) (Table 1).

\section{Response rate and survivals in the salvage therapy}

Conventional salvage therapy regimens, such as ESHAP ( $\mathrm{N}=93,38.4 \%)$, DHAP $(\mathrm{N}=9,3.4 \%)$, and ICE $(\mathrm{N}=14,5.8 \%)$ were performed. Meanwhile, R-containing therapies such as R-ESHAP ( $\mathrm{N}=63.26 .0 \%)$, R-DHAP $(\mathrm{N}=20,8.3 \%)$, and $\mathrm{R}-\mathrm{ICE}(\mathrm{N}=43,17.8 \%)$ were also performed. The conventional therapies did not differ in overall response rate (ORR) $(53.6 \%$ in ESHAP, $52.1 \%$ in DHAP, and 56.3\% in ICE, $P=0.417)$, 5-year PFS (33.2\% in ESHAP, $31.1 \%$ in DHAP, and $4.8 \%$ in ICE, $P=0.583$ ), and 5-year OS (38.1\% in ESHAP, $39.5 \%$ in DHAP, and $41.2 \%$ in ICE, $P=0.357)$. Moreover, there was no difference among the R-containing therapies (ORR, $74.3 \%$ in R-ESHAP, $72.4 \%$ in R-DHAP, and $76.2 \%$ in R-ICE, $P=0.365$; 5-year PFS, 43.4 in R-ESHAP, 42.7 in R-DHAP, and $44.1 \%$ in R-ICE, $P=0.397$; 5-year OS, $50.3 \%$ in R-ESHAP, $51.4 \%$ in R-DHAP, and $49.7 \%$ in R-ICE, $P=0.563$ ). However, ORR, 5-year PFS, and 5-year OS were significantly higher in the R-containing therapy than in the conventional therapy

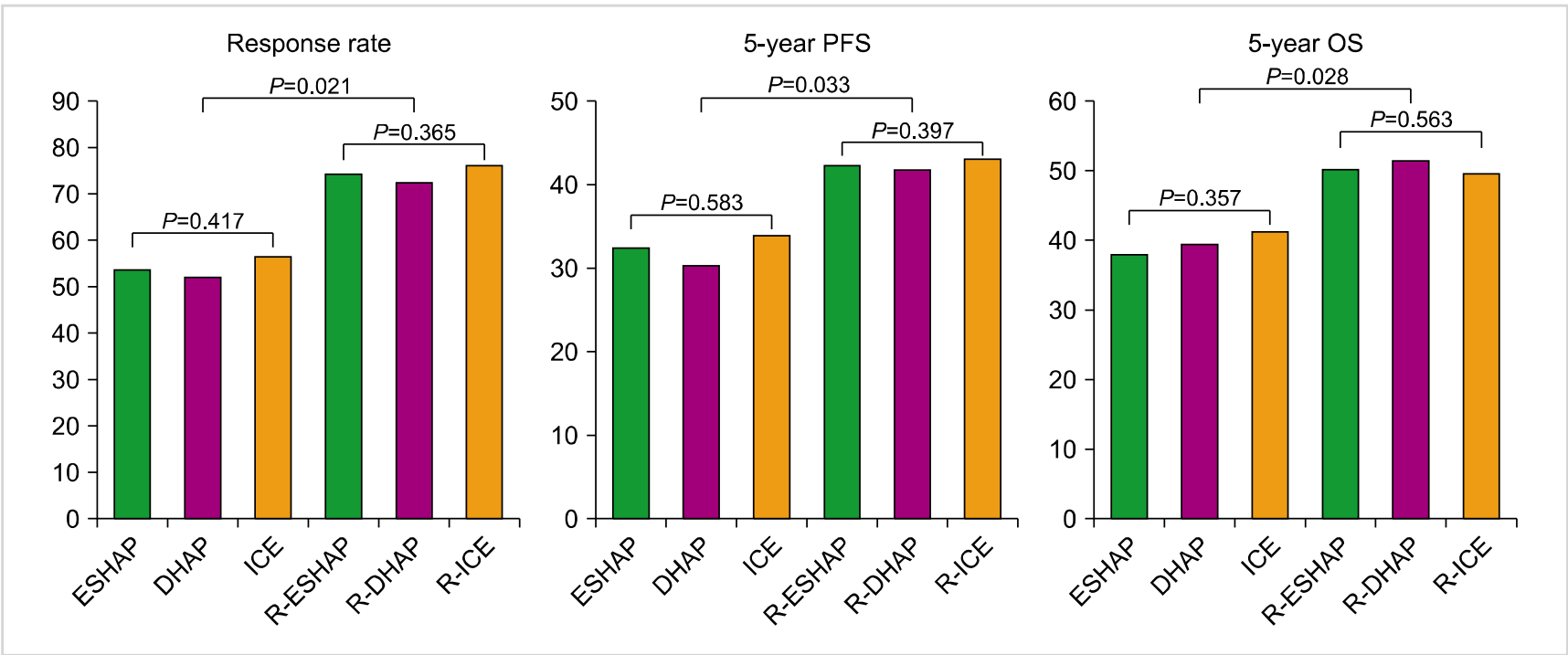

Fig. 1. Comparisons of the response rates and survivals among the salvage therapies administered to patients with relapsed diffuse large B cell lymphoma.

Table 2. Receiver operating characteristic analysis of continuous variables as prognostic factors for predicting disease progression in patients with relapsed diffuse large B cell lymphoma.

\begin{tabular}{lcccc}
\hline Prognostic markers & Cut-off value & Sensitivity $(\%)$ & Specificity (\%) & AUC $(95 \% \mathrm{Cl})$ \\
\hline NLR & 1.5 & 90.1 & 78.8 & $0.587(0.511-0.664)$ \\
dNLR & 3.5 & 90.4 & 81.2 & $0.767(0.706-0.829)$ \\
LMR & 1.6 & 90.6 & 73.2 & $0.687(0.618-0.757)$ \\
PLR & 100.0 & 89.4 & 81.1 & $0.641(0.570-0.712)$ \\
PNI & 133.0 & 90.6 & 70.7 & $0.611(0.538-0.685)$ \\
SUVmax & 10.0 & 88.5 & 81.6 & $0.634(0.560-0.709)$ \\
SII & 180.0 & 89.1 & 76.3 & $0.591(0.513-0.670)$ \\
\hline
\end{tabular}

Abbreviations: AUC, area under curve; $\mathrm{Cl}$, confidence interval; dNLR, derived neutrophil/lymphocyte ratio; LMR, lymphocyte/monocyte ratio; NLR, neutrophil/lymphocyte ratio; PLR, platelet/lymphocyte ratio; PNI, Prognostic Nutritional Index; SII, Systemic Inflammation-Immune Index; SUVmax, maximum standard uptake value. 
( $P=0.021, P=0.033$, and $P=0.028$, respectively) (Fig. 1).

ROC analysis for continuous variables as prognostic factors The patients were separated into favorable and unfavorable groups according to each optimal NLR, dNLR, LMR, PLR, PNI, SUXmax, and SII cut-off value determined by ROC analysis. The cut-off values for NLR, dNLR, LMR, PLR, PNI, SUVmax, and SII for disease progression were 1.5, 3.5, 1.6,

Table 3. Univariate analysis for prognostic factors in relapsed/refractory patients with diffuse large B cell lymphoma.

\begin{tabular}{|c|c|c|c|c|}
\hline & \multicolumn{2}{|c|}{ Progression-free survival } & \multicolumn{2}{|c|}{ Overall survival } \\
\hline & \multicolumn{2}{|c|}{ Univariate analysis } & \multicolumn{2}{|c|}{ Univariate analysis } \\
\hline & $\mathrm{HR}(95 \% \mathrm{Cl})$ & $P$ & $\mathrm{HR}(95 \% \mathrm{Cl})$ & $P$ \\
\hline High IPI score & $2.347(1.125-1.779)$ & $<0.001$ & $2.995(1.312-4.457)$ & 0.002 \\
\hline High NCCN-IPI & $2.243(1.541-6.124)$ & $<0.001$ & $2.652(1.299-5.998)$ & $<0.001$ \\
\hline Male sex & $1.023(0.847-2.101)$ & 0.828 & $1.109(0.899-1.602)$ & 0.398 \\
\hline High B2MG & $3.112(1.265-5.784)$ & 0.031 & $2.316(1.221-4.717)$ & 0.043 \\
\hline Primary refractory type & $2.991(1.198-5.454)$ & 0.002 & $3.001(1.873-5.441)$ & $<0.001$ \\
\hline GPS 2 & $4.801(1.981-6.121)$ & $<0.001$ & $3.663(1.455-5.812)$ & $<0.001$ \\
\hline Low NLR & $0.778(0.435-0.981)$ & 0.002 & $0.669(0.403-0.981)$ & 0.007 \\
\hline High dNLR & $3.887(1.917-6.634)$ & $<0.001$ & $2.983(1.352-5.754)$ & $<0.001$ \\
\hline Low LMR & $2.212(1.587-4.111)$ & 0.001 & $2.912(1.603-6.011)$ & 0.001 \\
\hline Low PLR & $2.875(1.137-5.173)$ & $<0.001$ & $3.136(1.232-5.534)$ & 0.003 \\
\hline Low PNI & $0.812(0.508-0.925)$ & 0.002 & $0.874(0.723-0.961)$ & 0.002 \\
\hline High SUVmax & $3.623(1.691-4.992)$ & 0.002 & $0.756(0.563-0.918)$ & 0.041 \\
\hline Low SIRI & $2.021(1.231-5.904)$ & $<0.001$ & $2.918(1.498-5.712)$ & $<0.001$ \\
\hline Low SII & $1.198(0.875-1.532)$ & 0.856 & $1.102(0.849-1.449)$ & 0.857 \\
\hline R-containing salvage therapy & $0.774(0.451-0.973)$ & 0.009 & $0.712(0.669-0.856)$ & 0.043 \\
\hline B symptoms & $1.221(0.879-1.925)$ & 0.741 & $1.116(0.941-1.325)$ & 0.657 \\
\hline ASCT & $0.621(0.365-0.898)$ & $<0.001$ & $0.521(0.399-0.895)$ & $<0.001$ \\
\hline
\end{tabular}

Abbreviations: ASCT, autologous stem cell transplantation; B2MG, beta-2-microglobulin; dNLR, derived neutrophi/lymphocyte count ratio; GPS, glasgow prognostic score; IPI, International Prognostic Index; LMR, lymphocyte/monocyte ratio; NCCN, National Comprehensive Cancer Network; NLR, neutrophil/lymphocyte count ratio; PLR, platelet/lymphocyte count ratio; PNI, Prognostic Nutritional Index; R, rituximab; SUVmax, maximum standardized uptake value.

Table 4. Multivariate analysis for prognostic factors in relapsed/refractory patients with diffuse large B cell lymphoma.

\begin{tabular}{|c|c|c|c|c|}
\hline & \multicolumn{2}{|c|}{ Progression-free survival } & \multicolumn{2}{|c|}{ Overall survival } \\
\hline & \multicolumn{2}{|c|}{ Multivariate analysis } & \multicolumn{2}{|c|}{ Multivariate analysis } \\
\hline & $\mathrm{HR}(95 \% \mathrm{Cl})$ & $P$ & $\mathrm{HR}(95 \% \mathrm{Cl})$ & $P$ \\
\hline High IPI score & $1.007(0.525-1.895)$ & 0.992 & $0.762(0.415-1.399)$ & 0.280 \\
\hline High NCCN-IPI & $2.836(1.425-5.643)$ & 0.003 & $2.928(1.415-5.649)$ & 0.003 \\
\hline High B2MG & $1.230(0.879-3.102)$ & 0.587 & $1.132(0.689-2.014)$ & 0.398 \\
\hline Primary refractory type & $1.343(0.839-2.150)$ & 0.220 & $1.828(0.941-2.878)$ & 0.409 \\
\hline GPS 2 & $2.435(1.285-3.222)$ & 0.002 & $2.621(1.131-3.815)$ & 0.002 \\
\hline Low NLR & $0.992(0.541-1.820)$ & 0.981 & $1.102(0.605-2.006)$ & 0.750 \\
\hline High dNLR & $2.452(1.535-3.916)$ & 0.002 & $2.542(1.208-3.664)$ & 0.005 \\
\hline Low LMR & $1.241(0.762-2.022)$ & 0.386 & $1.466(0.906-2.372)$ & 0.119 \\
\hline Low PLR & $1.875(0.921-2.782)$ & 0.341 & $1.752(0.846-2.413)$ & 0.294 \\
\hline Low PNI & $0.864(0.508-1.468)$ & 0.588 & $0.886(0.508-1.544)$ & 0.569 \\
\hline High SUVmax & $1.146(0.772-1.701)$ & 0.499 & $0.899(0.606-1.306)$ & 0.550 \\
\hline Low SIRI & $1.088(0.705-1.680)$ & 0.703 & $1.313(0.848-2.032)$ & 0.222 \\
\hline R-containing salvage therapy & $0.597(0.406-0.878)$ & 0.009 & $0.759(0.523-1.102)$ & 0.147 \\
\hline ASCT & $0.319(0.313-0.578)$ & 0.004 & $0.394(0.214-1.231)$ & 0.451 \\
\hline
\end{tabular}

Abbreviations: ASCT, autologous stem cell transplantation; B2MG, beta-2-microglobulin; dNLR, derived neutrophi/lymphocyte count ratio; GPS, glasgow prognostic score; IPI, International Prognostic Index; LMR, lymphocyte/monocyte ratio; NCCN, National Comprehensive Cancer Network; NLR, neutrophil/lymphocyte count ratio; PLR, platelet/lymphocyte count ratio; PNI, Prognostic Nutritional Index; R, rituximab; SUVmax, maximum standardized uptake value. 
$100.0,133.0,10.0$, and 180.0, respectively. In the ROC analysis, the area-under-the-curve (AUC) values of NLR, dNLR, LMR, PLR, PNI, SUVmax, and SII were 0.587, 0.767, 0.687, $0.641,0.611,0.634$, and 0.591 , respectively (Table 2).

\section{Clinical impacts of the prognostic factors}

Analyses to estimate the clinical impacts of several available prognostic factors were performed. The univariate analysis showed that high IPI (PFS, $P<0.001$; OS, $P=0.002$ ), high NCCN-IPI $(P<0.001 ; P<0.001)$, high B2MG $(P=0.031$; $P=0.043)$, the primary refractory type $(P=0.002 ; P<0.001)$, GPS $2(P<0.001 ; P=0.002)$, low NLR $(P=0.002 ; P=0.007)$, high dNLR $(P<0.001 ; P<0.001)$, low LMR $(P=0.001$; $P=0.001)$, low PLR $(P<0.001 ; P=0.003)$, low PNI $(P=0.002$; $P=0.002)$, high SUVmax $(P=0.002 ; P=0.041)$, low SIRI $(P<$ $0.001 ; P<0.001)$, the R-containing salvage therapy $(P=0.009$; $P=0.043)$, and ASCT $(P<0.001 ; P<0.008)$ were significantly associated with $\mathrm{PFS}$ and $\mathrm{OS}$ in patients with relapsed or refractory DLBCL (Table 3 ).

To evaluate the independent influence of each prognostic factor that was found significant by the univariate analysis, multivariate analysis was performed. High NCCN-IPI (PFS, $\mathrm{HR}=2.836,95 \% \mathrm{CI}=1.425-5.643, P=0.003 ; \mathrm{OS}, \mathrm{HR}=2.928$, 95\% CI=1.415-5.649, $P=0.003$ ), GPS 2 (PFS, HR=2.435, 95\% $\mathrm{CI}=1.285-3.222 ; \quad P=0.002 ; \mathrm{OS}, \mathrm{HR}=2.621,95 \% \mathrm{CI}=1.131-$ 3.815, $P=0.002$ ), and high dNLR (PFS, HR=2.452, 95\% $\mathrm{CI}=1.535-3.916, \quad P=0.002$; OS, HR=2.542, 95\% CI=1.2083.664, $P=0.005)$ were independently associated with both $\mathrm{PFS}$ and OS. However, the R-containing salvage therapy (PFS, HR=0.597, 95\% CI=0.406-0.878, $P=0.009$ ) and ASCT (PFS, HR=0.319, 95\% CI=0.313-0.578, $P=0.004$ ) were associated with only PFS (Table 4).

\section{Clinical outcomes according to NCCN-IPI, GPS, and dNLR as independent prognostic factors}

In the median follow-up period of 60.5 months (range, 2.6-142.1 mo), 5-year PFS and 5-year OS in low NCCN-IPI group were significantly higher than in high NCCN-IPI group (5-yr PFS $43.6 \%$ in low NCCN-IPI group vs. $11.1 \%$ in high NCCN-IPI group, $P<0.001$; and 5-yr OS, $39.1 \%$ in low NCCN-IPI group vs. $27.0 \%$ in high NCCN-IPI group, $P<0.001$, Fig. 2A, B). In addition, 5-year PFS and 5-year

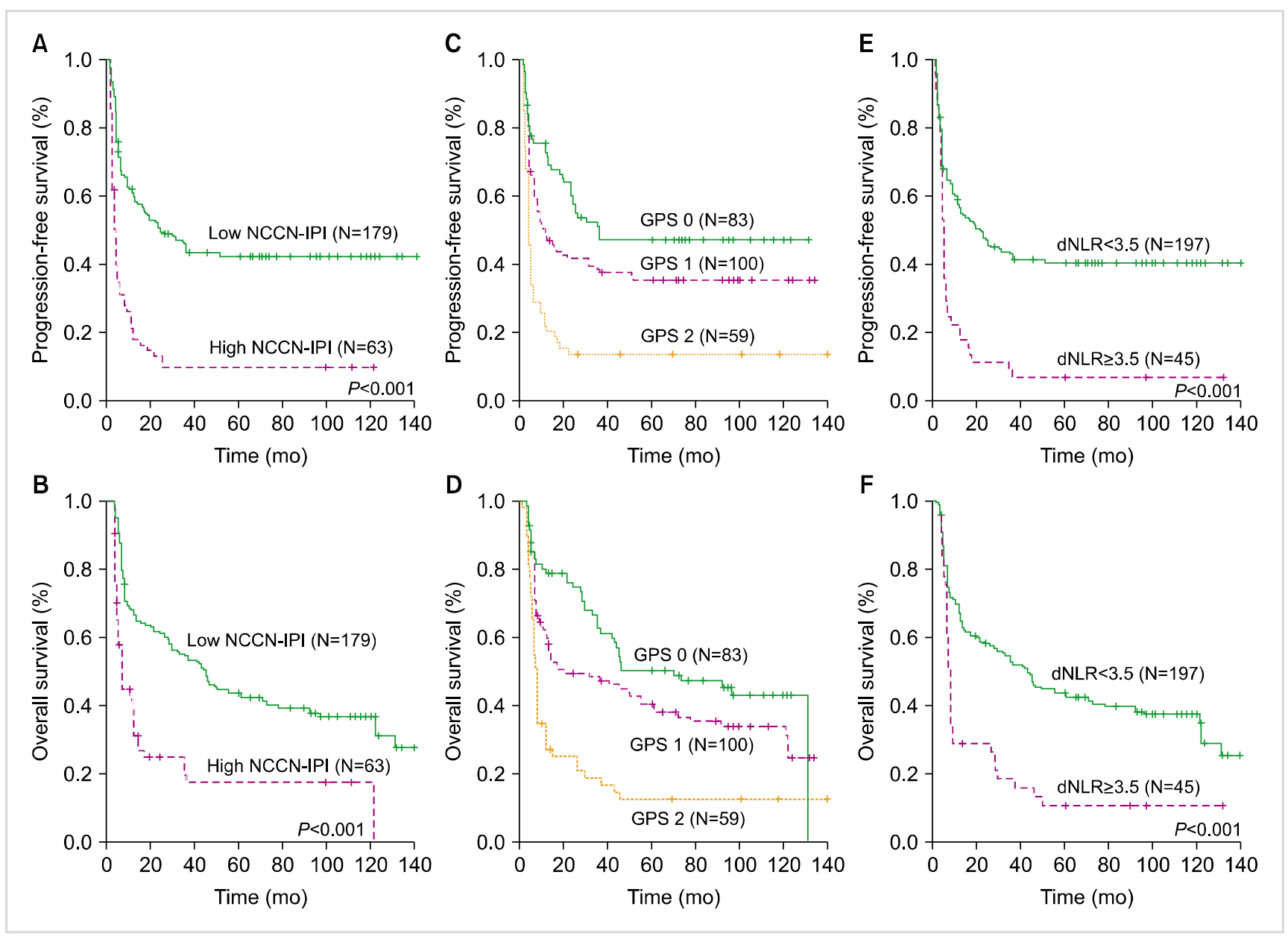

Fig. 2. Comparisons of the survival rates according to NCCN-IPI, glasgow prognostic score, and derived neutrophil/lymphocyte ratio in patients with relapsed diffuse large $B$ cell lymphoma.

Abbreviations: dNLR, derived neutrophil/lymphocyte ratio; GPS, glasgow prognostic score; NCCN-IPI, National Cancer Center Network-International Prognostic Index. 

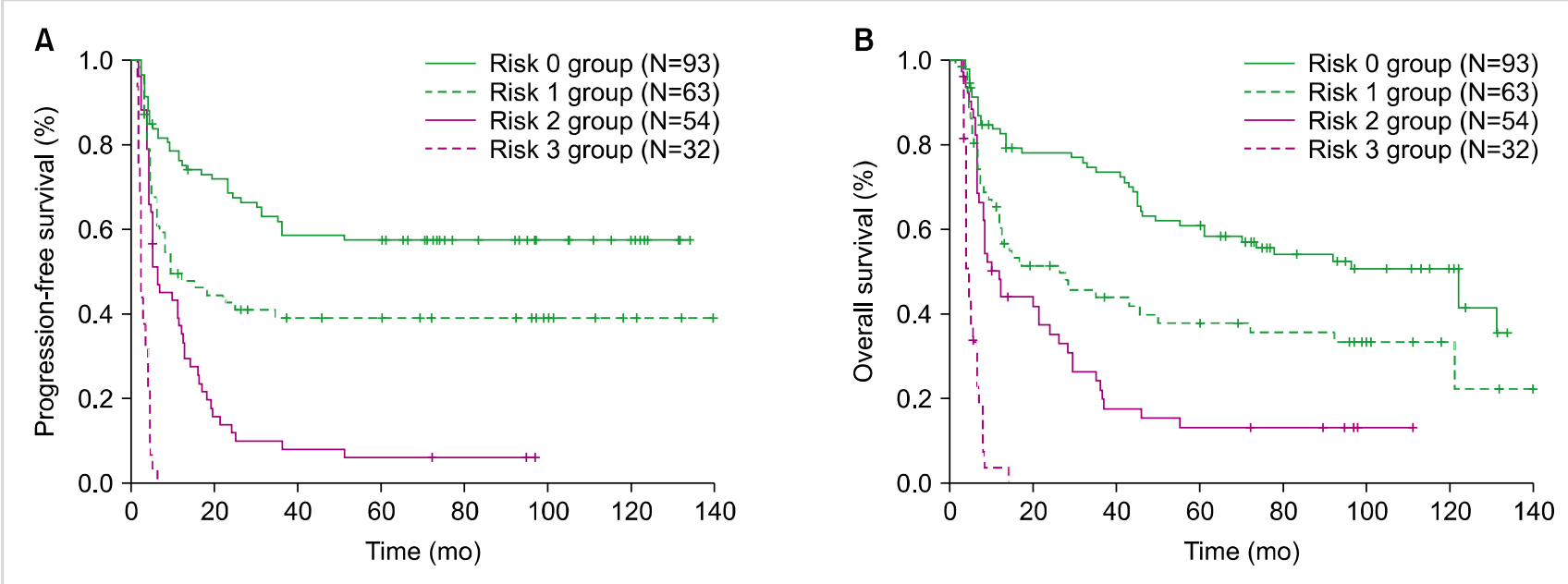

Fig. 3. Comparisons of survivals according to a combined scoring system based on NCCN-IPI, glasgow prognostic score, and derived neutrophil/lymphocyte ratio in patients with relapsed diffuse large B cell lymphoma.

OS according to GPS were significantly different (5-yr PFS, $49.4 \%$ in GPS $0,36.0 \%$ in GPS 1 , and $13.6 \%$ in GPS 2 , $P<0.001$; and 5 -yr OS, $48.2 \%$ in GPS $0,35.0 \%$ in GPS 1 , and $20.3 \%$ in GPS $2, P<0.001$, Fig. 2C, D). The survivals in low dNLR group were also higher than in high dNLR group (5-yr PFS, $41.6 \%$ in low dNLR group vs. $6.7 \%$ in high dNLR group, $P<0.001$; and 5-yr OS, $41.1 \%$ in dNLR group vs. $13.3 \%$ in high dNLR group, $P<0.001$, Fig. $2 \mathrm{E}$, F).

\section{Validation of the risk stratification model}

The risk stratification model was constructed using the independent prognostic factors, including high NCCN-IPI, GPS 2, and high dNLR obtained by the multivariate analysis. In the model, each factor was given the same point because of the similar HRs of the factors in the Cox proportional hazard model. Fig. 3 shows the stratification for validated risk based on NCCN-IPI, GPS, and dNLR to predict PFS and OS in patients with relapsed or refractory DLBCL. There were significant survival differences among the four risk groups (PFS, $P<0.001$; OS, $P<0.001$, Fig. 3 ).

\section{DISCUSSION}

To date, a risk stratification model that is based on clinical and laboratory parameters has not been proposed for patients with relapsed or refractory DLBCL. Although disease progression and survival in DLBCL could be determined by numerous factors, inflammation has an enhancing effect on malignant cell proliferation, angiogenesis, and metastasis.

Cell-based inflammation consisting of macrophages, neutrophils, monocytes, lymphocytes, and platelets is significantly associated with the progression of cancer and metastasis of malignant cells [21-23]. Moreover, several inflammatory cytokines produced by cancer cells, such as tumor necrosis factor (TNF)- $\alpha$, IL-1, IL-6, IL-8, and vascular endothelial growth factor also promote cancer cell proliferation, invasion, and metastasis [24]. NLR and dNLR have been repeatedly suggested to have prognostic associations with newly diagnosed DLBCL $[12,13]$. Here, we assessed for the clinical associations of these parameters with patients who had relapsed or refractory DLBCL and found in the multivariate analysis of our data that only dNLR, but not NLR, had a significant prognostic value. We suppose that neutrophil counts in patients with relapsed or refractory DLBCL are often unstable due to numerous factors, such as the influence of front-line chemotherapy, advanced disease status, and several concomitant comorbidities. Neutropenia can often be promptly corrected with the well-established supportive care practices, such as administration of recombinant granulocyte colony-stimulating factors. Because it is hard to decide the time point for the measurement of NLR, the clinical value of NLR is assumed less significant. However, neutrophil count is excluded in the assessment of dNLR even though dNLR seems to be more meaningful than NLR in the relapsed or refractory setting. Additionally, other cell-based inflammatory markers, including LMR, PLR, SIRI, and SII did not exhibit any statistical significance in our study. Furthermore, the nutrition-related inflammatory marker PNI did not have a significant value in the multivariate analysis.

It is likely that GPS, as a nutrition-related inflammatory marker consisting of serum CRP and albumin levels, respectively reflects the degree of cancer-related inflammation and the nutritional status. CRP, as a component of GPS, is an important and sensitive marker for systemic inflammatory response. The synthesis of CRP is generally induced by several cytokines, such as TNF- $\alpha$, IL-1, and IL-6 in the liver or cancer cells $[25,26]$. Therefore, the CRP level may conveniently reflect the degree of inflammation associated with cancer-related cytokines in a cancerous condition. This has been supported in several clinical studies that have reported that elevated CRP level is associated with 
poor prognosis in patients with various malignancies [27-31]. Increased serum albumin level is also considered an important sign of increased inflammation, impaired nutritional status, and other detrimental clinical conditions that result in a decreased therapeutic response rate, and thus tumor progression continues.

In the relapse or refractory setting, blood cell counts might be altered by previous chemotherapy or a systemic condition. Thus, GPS, which is not associated with blood cell counts, is possibly a more accurate prognostic factor than others, including blood cell counts. Moreover, GPS could reflect nutritional status in addition to cancer-related inflammation, unlike cell-based inflammatory markers. In our analyses, GPS was found to be an excellent predictive parameter for disease progression and survival in relapsed or refractory DLBCL patients.

In the present study, we investigated the clinical significance of IPI and NCCN-IPI as clinical prognostic factors in patients with relapsed or refractory DLBCL. Recent clinical studies have reported that NCCN-IPI is a better prognostic factor than conventional IPI in patients with newly diagnosed DLBCL [4]. However, clinical data in the relapsed or refractory setting is presumably still limited to confirm the predictive potential of NCCN-IPI. In our analyses, NCCN-IPI was also found to have a significant predictive potential for clinical outcomes in patients with relapsed or refractory DLBCL, but IPI was not.

To date, a validated prognostic model for patients with relapsed or refractory DLBCL has not been constructed. In this study, dNLR and GPS as inflammatory markers, and NCCN-IPI as a clinical factor, were found to influence the disease status and survival in the patients. We assessed whether the model of risk stratification could separate our patients into four significantly different risk groups. The results showed that our prognostic model could be offered as an alternative to the previously unorganized prognostic criteria in relapsed or refractory DLBCL. However, we did not analyze the gene expression profiles or immunohistochemistry data, because it was difficult to incorporate the COO subtype information obtained by these techniques into our retrospective study.

In conclusion, our study shows that dNLR, GPS, and NCCN-IPI meaningfully reflect disease progression and survival. Thus, these factors could be novel prognostic parameters for predicting outcomes in patients with relapsed or refractory DLBCL. Additionally, this is the first study that has attempted to delineate a novel risk stratification that incorporates an easily applicable inflammatory marker and clinical factor. However, our study has some limitations, such as low numbers of patients, retrospective design, and missing pathological data. To confirm our results, further clinical studies circumventing these issues are warranted.

\section{ACKNOWLEDGMENTS}

All authors significantly contributed to this study, and they all agree on the content of the manuscript.

\section{Authors' Disclosures of Potential Conflicts of Interest}

No potential conflicts of interest relevant to this article were reported.

\section{REFERENCES}

1. Morton LM, Wang SS, Devesa SS, Hartge P, Weisenburger DD, Linet MS. Lymphoma incidence patterns by WHO subtype in the United States, 1992-2001. Blood 2006;107:265-76.

2. Sehn LH, Donaldson J, Chhanabhai M, et al. Introduction of combined $\mathrm{CHOP}$ plus rituximab therapy dramatically improved outcome of diffuse large B-cell lymphoma in British Columbia. J Clin Oncol 2005;23:5027-33.

3. Gisselbrecht C, Glass B, Mounier N, et al. Salvage regimens with autologous transplantation for relapsed large B-cell lymphoma in the rituximab era. J Clin Oncol 2010;28:4184-90.

4. Zhou Z, Sehn LH, Rademaker AW, et al. An enhanced International Prognostic Index (NCCN-IPI) for patients with diffuse large B-cell lymphoma treated in the rituximab era. Blood 2014;123:837-42.

5. Prochazka KT, Posch F, Deutsch A, et al. Immunohistochemical double hit score enhances NCCN-IPI and is associated with detrimental outcomes in refractory or relapsing patients with diffuse large B cell lymphoma. Br J Haematol 2018;183:142-6.

6. Baecklund E, Iliadou A, Askling J, et al. Association of chronic inflammation, not its treatment, with increased lymphoma risk in rheumatoid arthritis. Arthritis Rheum 2006;54:692-701.

7. Loong F, Chan AC, Ho BC, et al. Diffuse large B-cell lymphoma associated with chronic inflammation as an incidental finding and new clinical scenarios. Mod Pathol 2010;23:493-501.

8. Sagaert X, Van Cutsem E, De Hertogh G, Geboes K, Tousseyn T. Gastric MALT lymphoma: a model of chronic inflammationinduced tumor development. Nat Rev Gastroenterol Hepatol 2010;7:336-46.

9. Richmond PC, Marshall HS, Nissen MD, et al. Safety, immunogenicity, and tolerability of meningococcal serogroup B bivalent recombinant lipoprotein 2086 vaccine in healthy adolescents: a randomised, single-blind, placebo-controlled, phase 2 trial. Lancet Infect Dis 2012;12:597-607.

10. Zhang L, Yang J, Qian J, et al. Role of the microenvironment in mantle cell lymphoma: IL-6 is an important survival factor for the tumor cells. Blood 2012;120:3783-92.

11. Marri PR, Hodge LS, Maurer MJ, et al. Prognostic significance of pretreatment serum cytokines in classical Hodgkin lymphoma. Clin Cancer Res 2013;19:6812-9.

12. Porrata LF, Ristow K, Habermann T, Inwards DJ, Micallef IN, Markovic SN. Predicting survival for diffuse large B-cell lymphoma patients using baseline neutrophil/lymphocyte ratio. Am J Hematol 2010;85:896-9.

13. Troppan K, Deutsch A, Gerger A, et al. The derived neutrophil to lymphocyte ratio is an independent prognostic factor in patients with diffuse large B-cell lymphoma. Br J Cancer 2014;110:369-74. 
14. Li ZM, Huang JJ, Xia Y, et al. Blood lymphocyte-to-monocyte ratio identifies high-risk patients in diffuse large B-cell lymphoma treated with R-CHOP. PLoS One 2012;7:e41658.

15. Sun F, Zhu J, Lu S, et al. An inflammation-based cumulative prognostic score system in patients with diffuse large B cell lymphoma in rituximab era. BMC Cancer 2018;18:5.

16. Hao X, Wei Y, Wei X, et al. Glasgow prognostic score is superior to other inflammation-based scores in predicting survival of diffuse large B-cell lymphoma. Oncotarget 2017;8:76740-8.

17. Li YL, Gu KS, Pan YY, Jiao Y, Zhai ZM. Peripheral blood lymphocyte/monocyte ratio at the time of first relapse predicts outcome for patients with relapsed or primary refractory diffuse large B-cell lymphoma. BMC Cancer 2014;14:341.

18. Jung SH, Yang DH, Ahn JS, Kim YK, Kim HJ, Lee JJ. Serum lactate dehydrogenase with a systemic inflammation score is useful for predicting response and survival in patients with newly diagnosed diffuse large B-cell lymphoma. Acta Haematol 2015;133:10-7.

19. Zhou $Q$, Wei $Y$, Huang F, et al. Low prognostic nutritional index predicts poor outcome in diffuse large B-cell lymphoma treated with R-CHOP. Int J Hematol 2016;104:485-90.

20. Cheson BD, Pfistner B, Juweid ME, et al. Revised response criteria for malignant lymphoma. J Clin Oncol 2007;25:579-86.

21. Lee SM, Russell A, Hellawell G. Predictive value of pretreatment inflammation-based prognostic scores (neutrophil-to-lymphocyte ratio, platelet-to-lymphocyte ratio, and lymphocyte-to-monocyte ratio) for invasive bladder carcinoma. Korean J Urol 2015;56: 749-55.

22. Kitayama J, Yasuda K, Kawai K, Sunami E, Nagawa H. Circulating lymphocyte number has a positive association with tumor response in neoadjuvant chemoradiotherapy for advanced rectal cancer. Radiat Oncol 2010;5:47.
23. Thomas MR, Storey RF. The role of platelets in inflammation. Thromb Haemost 2015;114:449-58.

24. Korniluk A, Koper O, Kemona H, Dymicka-Piekarska V. From inflammation to cancer. Ir J Med Sci 2017;186:57-62.

25. Castell JV, Gómez-Lechón MJ, David M, Fabra R, Trullenque R, Heinrich PC. Acute-phase response of human hepatocytes: regulation of acute-phase protein synthesis by interleukin-6. Hepatology 1990;12:1179-86.

26. Heikkilä K, Ebrahim S, Lawlor DA. A systematic review of the association between circulating concentrations of $\mathrm{C}$ reactive protein and cancer. J Epidemiol Community Health 2007;61:824-33.

27. Szkandera J, Gerger A, Liegl-Atzwanger B, et al. Validation of the prognostic relevance of plasma C-reactive protein levels in soft-tissue sarcoma patients. Br J Cancer 2013;109:2316-22.

28. Xiao WK, Chen D, Li SQ Fu SJ, Peng BG, Liang LJ. Prognostic significance of neutrophil-lymphocyte ratio in hepatocellular carcinoma: a meta-analysis. BMC Cancer 2014;14:117.

29. Szkandera J, Absenger G, Liegl-Atzwanger B, et al. Elevated preoperative neutrophil/lymphocyte ratio is associated with poor prognosis in soft-tissue sarcoma patients. Br J Cancer 2013;108: 1677-83.

30. Szkandera J, Gerger A, Liegl-Atzwanger B, et al. The lymphocyte/ monocyte ratio predicts poor clinical outcome and improves the predictive accuracy in patients with soft tissue sarcomas. Int J Cancer 2014;135:362-70.

31. Forrest LM, McMillan DC, McArdle CS, Angerson WJ, Dunlop DJ. Comparison of an inflammation-based prognostic score (GPS) with performance status (ECOG) in patients receiving platinumbased chemotherapy for inoperable non-small-cell lung cancer. Br J Cancer 2004;90:1704-6. 